\title{
Molecular Evolutionary Process of Advanced Gastric Cancer During Sequential Chemotherapy Detected by Circulating Tumor DNA
}

\section{Wenqi Xi}

Shanghai Jiao Tong University Medical School Affiliated Ruijin Hospital

Chenfei Zhou

Shanghai Jiao Tong University Medical School Affiliated Ruijin Hospital

Shouwei Zhang

Genecast Biotechnology Co., Ltd

Yi Zhao

Genecast Biotechnology Co., Ltd

Yawei Chen

Genecast Biotechnology Co., Ltd

Jun Ji

Shanghai Jiao Tong University Medical School Affiliated Ruijin Hospital

Tao Ma

Shanghai Jiao Tong University Medical School Affiliated Ruijin Hospital Junwei Wu

Shanghai Jiao Tong University Medical School Affiliated Ruijin Hospital Chengfang Shangguan

Shanghai Jiao Tong University Medical School Affiliated Ruijin Hospital

Zhenggang Zhu

Shanghai Jiao Tong University Medical School Affiliated Ruijin Hospital Jun Zhang ( $\square$ junzhang10977@sjtu.edu.cn )

Ruijin Hospital, Shanghai Jiaotong University School of Medicine

\section{Research}

Keywords: gastric cancer, sequential chemotherapy, dynamic ctDNA analysis, copy number variations, tumor evolution

Posted Date: September 1st, 2021

DOI: https://doi.org/10.21203/rs.3.rs-823245/v1 
License: (c) (i) This work is licensed under a Creative Commons Attribution 4.0 International License. Read Full License 


\section{Abstract}

Background: Chemotherapy is the major strategy for advanced gastric cancer (AGC) patients, whereits efficacy has largely plateaued. Tumor evolutionary theory provides a promising strategy to optimize paradigm of cancer treatment, while current data of molecular changes during sequential chemotherapy is too insufficient to understandevolutionary process in tumors.

Methods: Here, we performed NGS analysison 100 circulatingtumor DNA (ctDNA) samples collected from 27 AGC patients during treatment of sequentialchemotherapy. We observed dynamic changes of copy number instability $(\mathrm{CNI})$ and gene-level copy number variations (CNVs) during the treatment.

Results: Gene-levelCNV profiles were similar between baseline and end oftreatment. Subsequent regimens did not significantly change the $\mathrm{CNV}$ profiles driven by first-line regimens. Based on changes of copy number values of genes during treatment, resistance related genes of all four regimens were identified, respectively. These genes could be enriched into several common oncologic pathways, while exhibited a high inter-patient heterogeneity. Genes with different copy number values between baseline and end of treatment could be the targets of by molecular matched therapy.

Conclusions: Our study provides important information to understand molecular evolutionary process of AGS patients during sequential chemotherapy first and can help to optimize future treatment strategies.

\section{Background}

Gastric cancer is the third leading cause of cancer death worldwide. In 2020, more than 40\% of newly diagnosed cases of gastric cancer worldwide occurred in China, where the 5-year overall survival (OS) of Chinese patients was barely over $30 \%(1,2)$. Despite the rapid progression of molecular targeted therapy in the last decade, still multi-drug combination chemotherapy constitutes the backbone of therapy for most advanced gastric cancer (AGC) patients(3). Multiple clinical trials have been performed to optimize administration strategies of cytotoxic agents, while median overall survival of AGC patients is barely over 12 months.

Continuous administrating initial regimens which are commonly platinum-based doublet or triplet regimen until disease progression then subsequent regimens employed empirically is the traditional paradigm of AGC treatment. Nevertheless, multi-drug resistance is an inevitable problem under the continuous pressure of chemotherapy. The median PFS of AGC patients is about 6 to 8 months in firstline setting, while the median PFS of second-line (2L) chemotherapy is only about 2-4 months (4-6). The underlying molecular mechanisms playing in the chemotherapy resistance of AGC patients have been investigated based on bioinformatics and multi-omics analysis technologies by using surgical specimens, tumor biopsies and liquid biopsies(7-9). However, strategy to deal with treatment resistance of AGC in clinical practices is still lacking. 
Tumor that consists of highly heterogenous populations of tumor cells can be thought to be an open, complex ecosystem, and its phenotypes and behaviors can be partially explained by the Darwinian evolutionary theory. Resistant subpopulations of tumor cells can be selected during treatment of chemotherapy which is a drastic external pressure for the ecosystem of tumors. Meanwhile, to sustain treatment resistant phenotypes, fitness trade-offs as the cost of resistance can spontaneously occur in these subpopulations which may be vulnerable to other specific drugs. Therefore, tumor evolutionary process may provide a promising solution to modify or optimize current treatment paradigm of AGC patients and to improve patients' outcome.

Understanding molecular changes under the pressure of current treatment paradigm is necessary for oncologists to design a new treatment strategy based on tumor evolutionary theory. However, most investigations were mainly focused on the molecular changes that occur after treatment with one specific drug, chemotherapy regimen or molecular targeted agent, such as HER2 or PD-L1 antibodies(10-12). The molecular evolutionary process during sequential chemotherapy which is the most common treatment paradigm for Chinese AGC patients in clinical practices have not been fully delineated.

Consecutive sampling and analysis are necessary to elucidate the tumor evolutionary processes under the pressure of sequential treatment. Employment of liquid biopsy as a mini-invasive, dynamic and wellaccepted method for late-stage patients to detect molecular alterations in the tumor avoids the intratumoral heterogeneity and features high concordance with the results of the analysis of tumor lesions(13). Detection and analysis of circulating tumor DNA (ctDNA) using next generation sequencing are now widely employed for biomarker identification, minimal residual disease tracking, and treatment monitoring(14). Therefore, it is suitable for AGC patients to profile the molecular changes during sequential chemotherapy.

In the present study, peripheral blood samples were serially and prospectively collected from AGC patients who have been treated by typical chemotherapy regimens. Plasma ctDNA were analyzed utilizing a customized next generation sequencing (NGS) panel. Thus, our aim was to profile the molecular evolutionary process of AGC patients under the pressure of sequential chemotherapy.

\section{Materials And Methods}

\section{Patients and samples}

Peripheral blood samples were collected from gastric cancer patients who were treated in Department of Oncology, Ruijin Hospital and met the following criteria: 1) age over 18 years; 2) histologically confirmed HER2 negative stomach adenocarcinoma; 3) treatment naïve locally advanced, recurrent or metastasis disease; 4) adequate performance status and organ function to receive standard chemotherapy. Written informed consent was provided by all of the patients before collecting the peripheral blood samples. The protocol was approved by ethics committee of Ruijin Hospital, Shanghai Jiao Tong University School of Medicine, Shanghai, People's Republic of China. 
Blood samples were collected at times of baseline, efficacy assessment and disease progression. Samples collected at treatment failure of one regimen were considered as baseline samples for the followed regimen. Regimens with at least two samples during treatment were eligible for further analysis. Treatment efficacy was assessed by radiographic examination (contrast enhanced CT or MRI) following the routine clinical protocols. Objective response was assessed according to the RECIST criteria (version 1.1). Clinicopathological information of all of the patients was recorded, and PFS and OS were followed up.

\section{Dna Extraction, Sequencing And Data Processing}

Plasma and blood cells were separated by centrifugation at $1600 \mathrm{~g}$ for 10 minutes. Cell-free DNA (cfDNA) was isolated from plasma using MagMAX ${ }^{\mathrm{TM}}$ Cell-Free DNA Isolation Kit (Thermo Fisher Scientific, Waltham, MA, USA). Blood cell DNA was extracted from blood cells with TIANamp Blood DNA Kit (TIANGEN, Beijing, China). The concentration of DNA was measured by Qubit ${ }^{\circledR}$ dsDNA HS Assay Kit (Thermo Fisher Scientific, Waltham, MA, USA), and the quality of DNA was assessed by Agilent 2100 BioAnalyzer (Agilent, USA). Genomic DNA was sheared into 150-200 base-pair (bp) fragments with Covaris M220 Focused-ultrasonicator Instrument (Covaris, Massachusetts, USA). Fragmented DNA was used to construct the library using KAPA Hyper Preparation Kit (Kapa Biosystems, USA) according to the manufacturer's instruction. DNA was hybridized to one in-house panel (Genecast, Wuxi, China) covering $2.43 \mathrm{Mb}$ of the human genome with 1632 genes. The captured library was sequenced on the instrument of Illumina NovaSeq 6000 according to the manufacturer's instruction, producing paired-end reads with the length of each end as $150 \mathrm{bp}$.

Preliminary sequencing results in BCL format were converted to FASTQ files using bcl2fastq (v2.20.0). FASTQ format reads were processed using Trimmomatic (v0.39)(15) for adapter trimming and lowquality reads filtering. Processed reads were mapped to the reference genome (hg19) using BWA (0.7.17) (16). Mapped results were sorted and marked for duplications using Picard toolkit (version 2.1.0)(17), and then were realigned using GATK (version 3.7)(18).

\section{Somatic Snv Calling}

SNVs were called via VarDict (v1.5.1) and FreeBayes (v1.2.0) from processed mapping results in pair of tumor and control samples, respectively. The candidate somatic mutations were annotated with ANNOVAR(19) and then were filtered by ExAC, gnomAD, COSMIC, dbSNP databases. Nonsynonymous mutations among the exonic and splicing regions were kept for the final somatic mutation data set.

\section{Somatic Cnv Calling}


Taking blood cell sample as the control, somatic CNVs were called from plasma sample for each gene included in the captured panel using CNVkit (0.9.5.dev0) with batch mode(20). In the process of calling, biases of read depth, GC content and repetitive sequence were removed through normalization. The CNV value was defined as log2 transformed ratio of normalized read depth on each gene between plasma and blood cell samples.

\section{Calculation Of Tumor Mutation Burden (Tmb)}

TMB were determined by the nonsynonymous somatic mutations among the exonic and splicing regions with VAF (variant allele frequency) no less than $0.7 \%$. Alterations likely or known to be bona fide oncogenic drivers were excluded. TMB per megabase were calculated with the total number of mutations divided by the total bases of the target panel with $\geq 500 \mathrm{bp}$ coverage.

\section{Estimation Of Ctdna Content Fraction (Ccf)}

CCF of plasma samples was estimated by a maximum likelihood model based on SNVs and CNVs in the paired blood cell and plasma samples. Somatic and germline SNPs that met the following criteria were used to build the model: 1) with a minimum depth of 50x in the paired samples; 2) not on genes with high polymorphism; 3) no InDels in the 50bp upstream or downstream regions; 4) not in a copy number gain region; 5) germline SNPs with significantly different variant allele frequencies (VAFs) in the paired samples, or somatic SNPs with VAFs significantly higher than background noise. These SNPs were defined as informative SNPs and clustered into multiple groups according to their VAFs, local copy numbers and hypothetic genotypes. The hypothetic genotypes in cfDNA and ctDNA were determined by the VAFs in the paired samples and the copy number in the plasma sample. Each cluster represents a unique ctDNA source. We then calculated the likelihood of observing SNPs under given CCFs in each cluster. By maximizing the likelihood, CCF of each cluster could therefore be estimated. Cluster with the highest CCF was considered to be from the main source of ctDNA, and its CCF was the output of the final estimation.

\section{Estimation Of Copy Number Instability (Cni)}

After correction for GC content and length of target region using proprietary algorithms for each region, the read counts were transformed into log2 ratios and converted into Z-score based on Gaussian transformations versus a normal control group $(n=30)$. The target regions that satisfied the Z-score greater than the 95th percentile plus twice-times absolute standard deviation of the normal control group were retained, and these Z-scores were summed as the CNI score. 


\section{Screening For Sensitive And Resistant Genes Of Chemotherapy Drugs}

First, the ratio of copy number values of samples before and after treatment with chemotherapy drug was calculated for each gene. Here, only genes with positive or negative copy number values in both of the two samples were considered. Second, Drug Modified Score (DMS) of each gene was defined as log2 transformed of ratios calculated based on one pair of samples, as shown below:

$$
\text { DMS }=\log _{2} \frac{\text { Copy number value }}{\text { after treatment }}
$$

Third, genes were ranked based on DMS, and a curve of DMS versus gene rank was plotted for each drug (Fig. S2). Cutoffs of DMS for sensitive and resistant genes were determined utilizing the strategy of moving window with the window size of 5 genes(21). Along the window moving from smaller to larger DMS, the average slope of every 9 windows was calculated. When the average slope reached the slope of the middle point of the curve at the first time, DMS of the gene in middle of these 9 windows was considered as the cutoff of sensitive genes. Similarly, when the average slope reached the slope of middle point of the curve again, DMS of the gene in the middle of these 9 windows was considered as the cutoff of resistant genes. Considering that patient heterogeneity, resistant and sensitive genes were classified according to the rules which appeared more treatments for the resistant or the sensitive for each chemotherapy drugs. For Pt-2d and Pt-3d, one gene would be classified as resistant if three treatments had appeared and twice the opposite feature of treatments at least, and the same rule for sensitive genes. For PTX and CPT-11, one gene would be classified as resistant or sensitive if at least two treatments had appeared, and no treatment has appeared for the opposite feature. In total, we determined the resistant and the sensitive genes for four types of chemotherapy regimens. All of these analyses were conducted using an in-house script of R. Ten cancer pathways and 8 DNA repair pathways were curated for the enrichment analysis of resistant genes(22). For the major RTK RAS and PI3K pathways enriched, we described the SNV, CNV and the patient's percentage of the resistance among Pt-2d and Pt-3d.

\section{Statistical analysis}

Common, missing and new CNV events were analyzed between the baseline and the endpoint. Landscape of top 10 gene for each type were profiled by R package ComplexHeatmap(23). Significant genes between them were screened by Wilcoxon rank sum test using R. For significant genes, we screened and profiled resistant and sensitive features. To evaluate the molecular environment between EP1 and EP2, we selected genes that at least appeared in 3 samples in the baseline and the landscape was described by R package ComplexHeatmap. The heatmap of CNV value among these genes were also profiled. Boxplots of each gene were drawn using R packages. All other diagrams were also prepared using $\mathrm{R}$ packages.

\section{Results}




\section{Clinical characteristics of enrolled patients}

From June 2017 to January 2020, 27 patients were eligible for analysis. The clinical characteristics of enrolled patients are listed in Table 1. There were 18 males and 9 females, with a median age of 65 years (37 to 77 ). More than half of the patients (51.9\%) were gastric antrum origination. Fourteen cases had hematogenous metastasis. Four major chemotherapy regimens were identified, including platinum-based doublet regimen (Pt-2d), platinum-based triplet regimen (Pt-3d)(24), paclitaxel-based regimen (PTX) and irinotecan-based regimen (CPT-11). Fourteen patients were treated by Pt-3d and 13 patients were treated by Pt-2d as first-line regimen. Six patients and 5 patients were treated by CPT-11 and PTX as subsequent therapy, respectively. Therefore, two sequential treatment patterns, namely Pt-2d followed by PTX and Pt$3 \mathrm{~d}$ followed by CPT-11, were identified (Table S1). The objective response rate of the first-line treatment was $51.8 \%(14 / 27)$. At the end of follow-up (December 31, 2020), 3 patients were still alive. The median OS of these patients was 8.5 months and the progression free survival (PFS) of first-line setting was 4.7 months. 
Table 1

Clinical characteristics of 27 AGC patients

\begin{tabular}{|c|c|c|c|}
\hline \multicolumn{2}{|l|}{ Clinical characteristics } & \multirow{2}{*}{$\begin{array}{l}\text { Case (n) } \\
18\end{array}$} & \multirow{2}{*}{$\begin{array}{l}\text { Percentage (\%) } \\
66.7\end{array}$} \\
\hline Gender & Male & & \\
\hline & Female & 9 & 33.3 \\
\hline \multirow[t]{2}{*}{ Age } & Median & 65.0 & \\
\hline & Range & $37-77$ & \\
\hline \multirow[t]{3}{*}{ Tumor site } & Fundus & 5 & 18.5 \\
\hline & Body & 8 & 29.6 \\
\hline & Antrum & 14 & 51.9 \\
\hline \multirow[t]{2}{*}{ Hematogenous metastasis } & Yes & 14 & 51.9 \\
\hline & No & 13 & 48.1 \\
\hline \multirow[t]{2}{*}{ Signet cell } & Yes & 6 & 22.2 \\
\hline & No & 21 & 77.8 \\
\hline \multirow[t]{15}{*}{ Regimens } & Pt-3d & 14 & 51.9 \\
\hline & FLOT & 11 & / \\
\hline & DOS & 3 & / \\
\hline & Pt-2d & 13 & 48.1 \\
\hline & sox & 10 & / \\
\hline & CAPOX & 2 & / \\
\hline & FOLFOX & 1 & / \\
\hline & PTX & 5 & 18.5 \\
\hline & Paclitaxel & 2 & l \\
\hline & $\mathrm{PX}$ & 2 & / \\
\hline & Nab-Paclitaxel & 1 & / \\
\hline & $\mathrm{CPT}-11$ & 6 & 22.2 \\
\hline & IRIR & 3 & / \\
\hline & Irinotecan & 3 & / \\
\hline & FOLFIRI & 1 & / \\
\hline
\end{tabular}




\begin{tabular}{|llll|}
\hline Clinical characteristics & & Case (n) & Percentage (\%) \\
\hline Treatment pattern & Pt-3d to CPT-11 & 6 & 22.2 \\
\hline Pt-2d to PTX & 5 & 18.5 \\
\hline ORR of 1st-line & PR & 14 & 51.9 \\
& SD & 8 & 29.6 \\
\hline & PD & 5 & 18.5 \\
\hline
\end{tabular}

Correlation between genomic alterations in ctDNA and clinical efficacy of sequential chemotherapy

During the treatment, a total of 100 blood samples were collected prospectively and plasma ctDNA were extracted. The median sampling times for patients was 3 (2 to 10). Firstly, ctDNA content fraction (CCF), copy number instability (CNI) and tumor mutation burden (TMB) of all samples were calculated. The correlations between dynamic change of CCF and patients' survival time are illustrated in Fig. 1A. For most patients, CCF decreased from the baseline once the disease was under control, whereas it increased upon tumor progression. For patients with a relatively long-term disease control, CCF was also at a lowlevel. Dynamic change of CNI corresponded to the tendency of CCF (Fig. 1B).

Delta CNI defined as the difference between $\mathrm{CNI}$ at baseline and that in first sample after treatment was calculated to further verify the correlation between $\mathrm{CNI}$ and the treatment efficacy. For patients with partial response (PR) as their best overall response to the first-line regimen, the mean value of delta $\mathrm{CNI}$ was significantly lower than that in patients with stable disease (SD) or progression of disease (PD) ( $-1687.56 \pm 1617.54$ vs. $-147.71 \pm 1260.87, P=0.011$, Fig. 1 C). The correlations between CNI, CCF and TMB with treatment response were further illustrated in three typical cases (Fig. 1D). All these patients responded to first-line regimens while did not respond to subsequent regimens. After disease progression of initial regimen, $\mathrm{CNI}, \mathrm{CCF}$ and TMB all continued increasing during following subsequent treatment.

\section{Fluctuating Changes Of Gene-level Cnvs During Sequential Chemotherapy}

Gene-level copy number variants (CNVs) and single nucleotide variants (SNVs) were then detected utilizing a customized NSG panel including 1632 genes. For all of the samples, the top 10 recurrent genes with CNVs were NFKBIA, MCL 1, HSP9OAA1, MAP2K3, CREB1, PDPR, CALR, TOP1, IRS2 and PTPRT, and the top 10 recurrent genes with SNVs were TP53, LRP1B, CDH1, KMT2D, KMT2C, ARID1A, RHOA, KMT2B, $A P C$ and $C T N N B 1$ (Fig. 2, Fig. S1). A fluctuating change of gene-level CNVs could be observed from the landscape of all of the samples. Some gene CNVs were undetectable during the period of disease control while recurred at disease progression. 
To further delineate the fluctuating change of gene-level CNVs during sequential chemotherapy, the data of samples at baseline and endpoint of all 27 patients were compared first. Genes with CNVs could be assigned into three categories according to their changes during treatment comparing with baseline data, including genes that were recurrent at endpoint, those missing at endpoint and those specific at endpoint.

For each category, the top 10 genes are presented in Fig. 3A. Overlapping genes could be observed in the three categories, where the frequency of the recurrent and missing genes was higher than that of the endpoint specific genes. The majority of detected genes at the endpoint with high frequency were shared in recurrent and missing gene sets, including HSP9OAA1, CALR, NFKB1A, MCL 1 and CREB1. Genes that specifically occurred at the endpoint were only observed in a few patients. These genes included $N B N$, IRS2, LYN, SDHB and CSMD3.

\section{A subtle impact of subsequent regimens on gene-level CNVs driven by first-line treatment}

As the profiles of genes with CNVs were similar between baseline and endpoint of treatment, this result suggested that the effect of subsequent regimens on gene-level CNVs driven by first-line regimens might be subtle. There were 11 patients who received subsequent regimens in our cohort. Therefore, gene-level CNVs in ctDNA samples collected at endpoint of first-line regimen (EP1) and endpoint of second-line regimen (EP2) were analyzed. As was expected, most genes with CNVs were recurrent between EP1 and EP2 (Fig. 3B). EP2 specific genes with CNVs comparing with EP1 and baseline data occurred in only four patients (P17, P7, P25 and P8) including CALR, HSP90AA1, MYC, FAM135B, FGFR2 and CREB1.

Distribution of genes among these patients was highly heterogeneous, and no shared genes were identified among these patients.

\section{Significant difference of gene copy number values during sequential treatment}

Expect gene-level CNVs, copy number values of genes between the baseline and the endpoint were also analyzed. Unlike the CNVs, nineteen genes were identified to show significant differences. Genes with an increased copy number value after sequential treatment included CBFB, IL6ST, FBXW7, VEGFC, CYLD, FOXP1 and SMAD4. Genes with a decreased copy number value included FCRL1, UGT1A, SMARCD1, CASP8, PDCD1, CHST3, KMT5A, ATIC, ALK, RAD51D, NCOA1 and IKBKE (Fig. 4A).

Similar to the impact of subsequent regimens on gene-level CNVs, genes with significant different copy number values were not found between EP1 and EP2. For genes with CNVs detected in these samples, copy number value of genes was similar before and after the treatment of either PTX or CPT-11 as subsequent regimens (Fig. 4B).

\section{Resistance-related genes of chemotherapy regimens defined by drug modified score (DMS)}

The correlation between gene-level CNVs in ctDNA and treatment efficacy had been showed by our results. Therefore, we supposed that dynamic change of gene copy number values during treatment could be used to identified resistance-or sensitivity-related genes of chemotherapy regimens. DMS of genes was calculated based on dynamic changes of gene copy number values during treatment 
comparing with their baseline data. Resistance-related genes of each regimen were identified as those with increasing abnormality of copy number values (DMS increased) during treatment, while sensitivityrelated genes were identified as those with decreasing abnormality of copy number values (DMS decreased). There were 69 genes for Pt-2d, 86 genes for Pt-3d, 57 genes for PTX and 41 genes for CPT-11 identified as resistance-related genes (Fig. 5A, table S2). Genes with significant differential copy number value changes during treatment were also defined as resistance- or sensitivity-related genes in the four regimens employed (Fig. 5B).

Afterwards, the oncologic signaling pathway enrichment of the identified resistance-related genes was performed (Fig. 5C). Two major pathways, RTK RAS pathway and PI3K pathway, were identified in all four regimens. Homologous recombination pathway and TGF-beta pathway were shared between Pt-3d and PTX regimes. Nrf2 pathway and mismatch repair pathway were also related with tumor resistance to Pt$3 \mathrm{~d}$ regimen. Myc pathway was more specific to CPT-11 regimen and nucleotide excision repair pathway was specific to Pt-2d regimen.

\section{A high inter-patient heterogeneity of involved resistance-related genes in common enriched pathways}

Afterwards, we turned our focus on two common pathways, namely RTK RAS pathway and PI3K pathway. Based on the data of Pt-2d and Pt-3d regimens, the resistance-related genes in play have been labeled on the diagrams of RTK RAS pathway (Fig. 6A) and PI3K pathway (Fig. 6B) according to the types of genomic alterations detected at the baseline and their frequency in patients. CNV gain was observed in most of the oncogenes including PIK3CA, AKT, MTOR, EGFR family, FGFR and RET, where CNV loss was observed in tumor suppressor genes including PTEN, STK11, TSC1, PIK3R and PTPN11. For patients who were treated by Pt-3d $(n=14)$ or Pt-2d $(n=13)$, almost all of the genes in these two pathways could be identified as resistance-related genes, where the proportion of patients who carried those genes was relatively low. Most of such genes occurred in less than $30 \%$ of patients, which showed a high interpatient heterogeneity.

\section{Discussion}

In the present study, the dynamic change of gene-level CNVs in ctDNA during sequential chemotherapy has been analyzed in the peripheral blood samples serially collected from AGC patients. Gene-level CNV profiles were highly consistent before and after the treatment in all of the patients, and no significant impact of subsequent regimes on the molecular changes that were driven by first-line regimens was observed. Under the pressure of four different chemotherapy regimens, genes that were defined as resistance-related according to their dynamic change of copy number values during treatment could be enriched into several common oncologic signaling pathways, where those genes exhibited a high heterogeneity among patients.

Tumor evolutionary theory provides an alternative strategy to optimize current treatment paradigm of advanced or metastatic malignant tumors(25). During tumor adaptation to drastic external pressure, fitness trade-offs occur spontaneously as the cost of evolution which result in vulnerable of tumor cells to 
selected subsequent therapy $(21,26,27)$. This phenomenon provides a probability for oncologists to anticipate tumor evolutionary process under the pressure of certain drugs and design an optimal subsequent regimen to prolong disease control(28), which can be completely different from current treatment paradigms of AGC patients. However, to employ this strategy, molecular evolutionary process during whole course of treatment should be understood first, then a reliable surveillance method is also necessary. In present study, we first delineated the dynamic change of gene-level CNVs during sequential chemotherapy of AGC patients by ctDNA analysis in serially peripheral blood samples and verified the feasibility of ctDNA analysis to monitor molecular evolutionary process of AGC.

Reviewing the landscape of genomic alterations detected in present study, it was found that genes carrying CNVs and SNVs with high frequency have also been reported in the TCGA and ACRG cohorts of gastric cancer, for which, comprehensive molecular analysis have been performed based on tumor tissues $(29,30)$. The common genes with CNVs reported by TCGA and ACRG cohorts, such as EGFR, FGFR2, MYC and CCND1, have also been detected in our cohort. TP53 is the most frequent mutated gene, and other common mutated genes in gastric cancer, including CHD1, KMT2C, ARID1A, RHOA, and CTNNB1, have been detected in our ctDNA analysis. Our data demonstrated that the customized NSG panel that we used to detect molecular changes in ctDNA samples was reliable to analyze intratumoral molecular alterations.

In previous investigations, ctDNA analysis during treatment has been performed and was mainly focused on the quantitive data. The dynamic changes of TMB, CNI and CCF in our data were similar with reported results, which decreased during disease control and increased after disease progression(31). By using the customized NSG panel, we could further analyze the qualitative data. A pan-cancer study demonstrated that there was a positive linear influence of CNVs on the expression of most of the genes, which indicated a direct effect of CNVs on gene transcriptional level(32). And also, gene CNVs are considered as very common and important molecular alterations in tumors and are usually the upstream changes that trigger the cascade of signaling chaos(33). Therefore, we focused our study on the gene-level CNVs during sequential treatment.

A fluctuating change of gene CNVs could be observed on the landscape of all of the samples.

Furthermore, common molecular profiles were found at baseline and endpoint, as well as at EP1 and EP2, which suggested that the treatment failure of sequential chemotherapy in AGC was partially due to regrowth of tumor clones that preexisted at the baseline rather than the new subclones that were driven by treatment pressure. Employment of commonly used subsequent chemotherapy regimens, such as paclitaxel-based and irinotecan-based, could not significantly change in the profiles of gene-level CNVs that were driven by the pressure of first-line regimes. Paclitaxel or irinotecan monotherapy are both the standard $2 \mathrm{~L}$ regimens for AGC patients. The median PFS of these two regimens was found to be only about 3 months and ORR was about 10-20\%(6). So far, the underlying molecular mechanisms in play for the poor efficacy of cytotoxic agents in $2 \mathrm{~L}$ setting of AGC patients have not been focused. Our result provided an explanation about the poor efficacy of subsequent chemotherapy of AGC patients from molecular level. 
Tumor cells can adapt to external treatment pressure by acquisition of new genomic or epigenomic changes(34). At the same time, the dynamics of tumor evolution should be taken into account. An investigation into the genomic landscape of relapsed acute lymphoblastic leukemia showed that the prevalence of relapse-specific somatic alterations in patients who exhibited very early relapse ( $<9$ months from diagnosis) was significantly less than that of the patients who showed an early or late relapse(35). The median PFS of AGC patients in the first-line treatment is normally 6 to 8 months. Although in different kinds of tumors, these results suggested that there might not be long enough time for gastric cancer cells to acquire new driver somatic alterations under treatment pressure. Li, et al. also reported that CNV profiles were quite similar in paired tissue samples collected before and after neoadjuvant chemotherapy from gastric cancer patients who had no response (36).

During the adaptation of tumor cells to external pressure, many genes simply changed their expression rather than an "all or none" phenomenon $(37,38)$. Thus, the dynamic change of copy number values of genes was also important evolutionary information. Several cancer related genes with significant different copy number values between baseline and endpoint were identified, which verified this theory. VEFGC and $C Y L D$ that play roles in the angiogenesis process $(39,40)$ have been identified as genes with increased copy number value, and FOXP1, a member the forkhead box (FOX) transcription factor family, has been reported to be involved in the regulation of gastric cancer proliferation(41). Conversely, CASP 8 , $P D C D 1$ and RAD51D have been identified as genes with decreased copy number values. The low expression of PD-1 on tumor cells promoted tumor cell proliferation (42). RAD51D, which plays a role in the break repair of the double-stranded DNA, has also been associated with sensitivity to platinum treatment(43). However, differential copy number values of genes were not identified at EP1 and EP2, which further verified the subtle impact of current subsequent regimens on the molecular evolution driven by first-line treatment in AGC patients. Several molecular targeted agents, including ramucirumab, apatinib, immune checkpoint inhibitors (ICls), showed efficacy in AGC patients as subsequent regimens. The changes of copy number values of VEGFC, CYLD and PDCD1 driven by the first-line treatment might provide a clue for applications of those drugs, as ramucirumab and apatinib are both anti-angiogenetic agents as well as ICls targeting PD-1 (7, 44, 45).

Using the dynamic data of gene copy number values during treatment, resistance-related genes were identified for each regimen. A convergent evolution-like process was found(46). Several common pathways, including RTK RAS pathway and PI3K pathway, could be enriched among all regimens by using resistance-related genes. Aberration of oncological pathways regulating important tumor behaviors, including sustained proliferation, cell death evasion and angiogenesis, is also closely correlated with chemoresistance of tumor cells(47). Therefore, these shared pathways might be attributed to be the underlying mechanisms of treatment failure and could also be the potential targets in subsequent treatment.

Molecular matched therapy had been performed in clinical trials based on molecular alterations detected in tumors. SHIVA trial was a randomized phase II trial assessing molecular matched therapy in refractory solid tumors(48). VIKTORY umbrella trial enrolled and classified AGC patients who were offered to 
undergo second-line treatment into eight different biomarker groups according to clinical sequencing(49). However, SHIVA trial did not show significant benefit, while absolute time of survival extension in VIKTORY trial, a non-randomized trial, was only about 2 months. From our results, the genes involved in these pathways were found highly heterogeneous among patients. It is inferred that while common aberrant pathways might result in treatment failure, inter-patient heterogeneity and signaling crosstalk could result in inefficacy of agents targeting single molecular(50). Therefore, a multi-target strategy should be employed as subsequent regimens of AGC patients. Results from several trials have provided some evidence to support this phenomenon. In EPOC1706 trial, lenvatinib plus pembrolizumab in the first-line or $2 \mathrm{~L}$ setting of ACG patients showed $69 \%$ of objective response rate, which was much higher than any other regimens that were administrated in $2 \mathrm{~L}$ setting of AGC patients before(51). REGONIVO trial also showed a high response rate for the treatment of refractory AGC patients with regorafenib plus nivolumab (52). In addition to the synergistic effects between these drugs and ICls, it should be highlighted that both lenvatinib and regorafenib are multi-targeted tyrosine kinase inhibitors of VEGFR, FGFR, PDGFR, KIT and RET $(53,54)$.

The present study possesses certain limitations. First, the fact that 27 patients were enrolled in our study made the sample size relatively small. Nevertheless, analysis of $100 \mathrm{ctDNA}$ samples could give a representative view over the dynamic molecular changes during the treatment. Second, gene CNVs of subsequent therapy were only analyzed in eleven patients. The fact that the available information of the literature regarding the dynamic molecular changes during sequential chemotherapy of AGC patients is limited gives importance to the results of our study. Third, although differential copy number values of genes were identified in our study, correlations between them and the gene expression were not investigated. Thus, it is our aim to investigate the correlations between differential gene copy number values and transcriptional data in future studies using a larger sample size.

\section{Conclusions}

In summary, dynamic analysis of somatic gene-level CNVs of ctDNA in peripheral blood can be effectively used to monitor tumor evolutionary process under pressure of sequential chemotherapy. After treatment of standard chemotherapy regimens, a convergent oncologic pathway enrichment and yet inter-patient heterogenous gene-level CNVs was observed, which provided important information to optimized molecular matched therapies for sequential treatment of AGC patients.

\section{Abbreviations}

OS, overall survival; AGC, advanced gastric cancer; ctDNA, circulating tumor DNA; NGS, next generation sequencing; TMB, tumor mutation burden; VAF, variant allele frequency; CCF, ctDNA content fraction; CNI, copy number instability; PR, partial response; SD, stable disease;

PD, progression of disease; CNVs, copy number variants; SNVs, single nucleotide variants; DMS, drug modified score; ICls, immune checkpoint inhibitors. 


\section{Declarations}

\section{Ethics approval and consent to participate}

Written informed consent was provided by all of the patients before collecting the peripheral blood samples. The protocol was approved by ethics committee of Ruijin Hospital, Shanghai Jiao Tong University School of Medicine, Shanghai, People's Republic of China.

\section{Consent for publication}

Not applicable

\section{Availability of data and materials}

All data generated or analyzed during this study are included in this paper.

\section{Competing interests}

The authors declare that they have no competing interests.

\section{Funding}

The study was supported by National Science Foundation of China (81972707 and 81802319)

\section{Author contributions}

Z.J. and X.WQ. conceived and designed the research. X.WQ., Z.CF., J.J., M.T., W.JW. and SG.CF. contributed to the collection of specimens, radiological evaluation and discussion of clinical significance. Z.J. and Z.CF. contributed to design of the analysis. Z.SY., C.YW. and Z.Y led the data analysis. Z.SY. and Z.CF. contributed to the data analysis. Z.J., Z.CF and Z.SW. wrote the manuscript. Z.J. supervised the whole project.

\section{Acknowledgments}

Not applicable

\section{References}

1. Chen W, Zheng R, Baade PD, Zhang S, Zeng H, Bray F, et al. Cancer statistics in China, 2015. CA: a cancer journal for clinicians. 2016;66(2):115-32.

2. Gao K, Wu J. National trend of gastric cancer mortality in China (2003-2015): a population-based study. Cancer communications. 2019;39(1):24.

3. Joshi SS, Badgwell BD. Current treatment and recent progress in gastric cancer. CA: a cancer journal for clinicians. 2021;71(3):264-79. 
4. Cunningham D, Starling N, Rao S, Iveson T, Nicolson M, Coxon F, et al. Capecitabine and oxaliplatin for advanced esophagogastric cancer. The New England journal of medicine. 2008;358(1):36-46.

5. Ajani JA, Rodriguez W, Bodoky G, Moiseyenko V, Lichinitser M, Gorbunova V, et al. Multicenter phase III comparison of cisplatin/S-1 with cisplatin/infusional fluorouracil in advanced gastric or gastroesophageal adenocarcinoma study: the FLAGS trial. J Clin Oncol. 2010;28(9):1547-53.

6. Hironaka S, Ueda S, Yasui H, Nishina T, Tsuda M, Tsumura T, et al. Randomized, open-label, phase III study comparing irinotecan with paclitaxel in patients with advanced gastric cancer without severe peritoneal metastasis after failure of prior combination chemotherapy using fluoropyrimidine plus platinum: WJOG 4007 trial. J Clin Oncol. 2013;31(35):4438-44.

7. Nagaraja AK, Kikuchi O, Bass AJ. Genomics and Targeted Therapies in Gastroesophageal Adenocarcinoma. Cancer discovery. 2019;9(12):1656-72.

8. Oh SC, Sohn BH, Cheong JH, Kim SB, Lee JE, Park KC, et al. Clinical and genomic landscape of gastric cancer with a mesenchymal phenotype. Nature communications. 2018;9(1):1777.

9. Sa JK, Hong JY, Lee IK, Kim JS, Sim MH, Kim HJ, et al. Comprehensive pharmacogenomic characterization of gastric cancer. Genome medicine. 2020;12(1):17.

10. Kim ST, Cristescu R, Bass AJ, Kim KM, Odegaard JI, Kim K, et al. Comprehensive molecular characterization of clinical responses to PD-1 inhibition in metastatic gastric cancer. Nature medicine. 2018;24(9):1449-58.

11. Pietrantonio F, Fuca G, Morano F, Gloghini A, Corso S, Aprile G, et al. Biomarkers of Primary Resistance to Trastuzumab in HER2-Positive Metastatic Gastric Cancer Patients: the AMNESIA CaseControl Study. Clinical cancer research : an official journal of the American Association for Cancer Research. 2018;24(5):1082-9.

12. Zhou C, Feng X, Yuan F, Ji J, Shi M, Yu Y, et al. Difference of molecular alterations in HER2-positive and HER2-negative gastric cancers by whole-genome sequencing analysis. Cancer management and research. 2018;10:3945-54.

13. Kilgour E, Rothwell DG, Brady G, Dive C. Liquid Biopsy-Based Biomarkers of Treatment Response and Resistance. Cancer cell. 2020;37(4):485-95.

14. Alix-Panabieres C, Pantel K. Liquid Biopsy: From Discovery to Clinical Application. Cancer discovery. 2021;11(4):858-73.

15. Bolger AM, Lohse M, Usadel B. Trimmomatic: a flexible trimmer for Illumina sequence data. Bioinformatics. 2014;30(15):2114-20.

16. Li H, Durbin R. Fast and accurate short read alignment with Burrows-Wheeler transform. Bioinformatics. 2009;25(14):1754-60.

17. Picard toolkit. Broad Institute, GitHub repository. 2019.

18. Van der Auwera GA, Carneiro MO, Hartl C, Poplin R, Del Angel G, Levy-Moonshine A, et al. From FastQ data to high confidence variant calls: the Genome Analysis Toolkit best practices pipeline. Curr Protoc Bioinformatics. 2013;43:11 0 1- 033. 
19. Wang K, Li M, Hakonarson H. ANNOVAR: functional annotation of genetic variants from highthroughput sequencing data. Nucleic acids research. 2010;38(16):e164.

20. Talevich E, Shain AH, Botton T, Bastian BC. CNVkit: Genome-Wide Copy Number Detection and Visualization from Targeted DNA Sequencing. PLoS computational biology. 2016;12(4):e1004873.

21. Lin KH, Rutter JC, Xie A, Pardieu B, Winn ET, Bello RD, et al. Using antagonistic pleiotropy to design a chemotherapy-induced evolutionary trap to target drug resistance in cancer. Nature genetics. 2020;52(4):408-17.

22. Sanchez-Vega F, Mina M, Armenia J, Chatila WK, Luna A, La KC, et al. Oncogenic Signaling Pathways in The Cancer Genome Atlas. Cell. 2018;173(2):321-37 e10.

23. Gu Z, Eils R, Schlesner M. Complex heatmaps reveal patterns and correlations in multidimensional genomic data. Bioinformatics. 2016;32(18):2847-9.

24. Zhou C, Ma T, Shi M, Xi W, Wu J, Yang C, et al. Dose-finding study of modified FLOT (mFLOT) regimen as first-line treatment in Chinese patients with metastatic adenocarcinoma of stomach. Cancer chemotherapy and pharmacology. 2020;85(1):113-9.

25. Enriquez-Navas PM, Wojtkowiak JW, Gatenby RA. Application of Evolutionary Principles to Cancer Therapy. Cancer research. 2015;75(22):4675-80.

26. Hausser J, Alon U. Tumour heterogeneity and the evolutionary trade-offs of cancer. Nature reviews Cancer. 2020;20(4):247-57.

27. Wang L, Leite de Oliveira R, Huijberts S, Bosdriesz E, Pencheva N, Brunen D, et al. An Acquired Vulnerability of Drug-Resistant Melanoma with Therapeutic Potential. Cell. 2018;173(6):1413-25 e14.

28. Stankova K, Brown JS, Dalton WS, Gatenby RA. Optimizing Cancer Treatment Using Game Theory: A Review. JAMA oncology. 2019;5(1):96-103.

29. Cancer Genome Atlas Research N. Comprehensive molecular characterization of gastric adenocarcinoma. Nature. 2014;513(7517):202-9.

30. Cristescu R, Lee J, Nebozhyn M, Kim KM, Ting JC, Wong SS, et al. Molecular analysis of gastric cancer identifies subtypes associated with distinct clinical outcomes. Nature medicine. 2015;21(5):449-56.

31. Parikh AR, Mojtahed A, Schneider JL, Kanter K, Van Seventer EE, Fetter IJ, et al. Serial ctDNA Monitoring to Predict Response to Systemic Therapy in Metastatic Gastrointestinal Cancers. Clinical cancer research : an official journal of the American Association for Cancer Research. 2020;26(8):1877-85.

32. Shao X, Lv N, Liao J, Long J, Xue R, Ai N, et al. Copy number variation is highly correlated with differential gene expression: a pan-cancer study. BMC medical genetics. 2019;20(1):175.

33. Hehir-Kwa JY, Pfundt R, Veltman JA. Exome sequencing and whole genome sequencing for the detection of copy number variation. Expert review of molecular diagnostics. 2015;15(8):1023-32.

34. McGranahan N, Swanton C. Clonal Heterogeneity and Tumor Evolution: Past, Present, and the Future. Cell. 2017;168(4):613-28. 
35. Li B, Brady SW, Ma X, Shen S, Zhang Y, Li Y, et al. Therapy-induced mutations drive the genomic landscape of relapsed acute lymphoblastic leukemia. Blood. 2020;135(1):41-55.

36. Li Z, Gao X, Peng X, May Chen MJ, Li Z, Wei B, et al. Multi-omics characterization of molecular features of gastric cancer correlated with response to neoadjuvant chemotherapy. Science advances. 2020;6(9):eaay4211.

37. Gatenby RA. A change of strategy in the war on cancer. Nature. 2009;459(7246):508-9.

38. Wind NS, Holen I. Multidrug resistance in breast cancer: from in vitro models to clinical studies. International journal of breast cancer. 2011;2011:967419.

39. Gao J, Sun L, Huo L, Liu M, Li D, Zhou J. CYLD regulates angiogenesis by mediating vascular endothelial cell migration. Blood. 2010;115(20):4130-7.

40. Yamaguchi T, Fushida S, Yamamoto Y, Tsukada T, Kinoshita J, Oyama K, et al. Tumor-associated macrophages of the $\mathrm{M} 2$ phenotype contribute to progression in gastric cancer with peritoneal dissemination. Gastric cancer : official journal of the International Gastric Cancer Association and the Japanese Gastric Cancer Association. 2016;19(4):1052-65.

41. Chen X, Liu C, Ji L, Wang N, Liu Y, Wang M, et al. The circACC1/miR-29c-3p/FOXP1 network plays a key role in gastric cancer by regulating cell proliferation. Biochemical and biophysical research communications. 2021;557:221-7.

42. Wang $X$, Yang $X$, Zhang $C$, Wang $Y$, Cheng $T$, Duan $L$, et al. Tumor cell-intrinsic PD-1 receptor is a tumor suppressor and mediates resistance to PD-1 blockade therapy. Proceedings of the National Academy of Sciences of the United States of America. 2020;117(12):6640-50.

43. Pennington KP, Walsh T, Harrell MI, Lee MK, Pennil CC, Rendi MH, et al. Germline and somatic mutations in homologous recombination genes predict platinum response and survival in ovarian, fallopian tube, and peritoneal carcinomas. Clinical cancer research : an official journal of the American Association for Cancer Research. 2014;20(3):764-75.

44. Fuchs CS, Doi T, Jang RW, Muro K, Satoh T, Machado M, et al. Safety and Efficacy of Pembrolizumab Monotherapy in Patients With Previously Treated Advanced Gastric and Gastroesophageal Junction Cancer: Phase 2 Clinical KEYNOTE-059 Trial. JAMA oncology. 2018;4(5):e180013.

45. Wilke H, Muro K, Van Cutsem E, Oh SC, Bodoky G, Shimada Y, et al. Ramucirumab plus paclitaxel versus placebo plus paclitaxel in patients with previously treated advanced gastric or gastrooesophageal junction adenocarcinoma (RAINBOW): a double-blind, randomised phase 3 trial. The Lancet Oncology. 2014;15(11):1224-35.

46. Misale S, Di Nicolantonio F, Sartore-Bianchi A, Siena S, Bardelli A. Resistance to anti-EGFR therapy in colorectal cancer: from heterogeneity to convergent evolution. Cancer discovery. 2014;4(11):1269-80.

47. Delou JMA, Souza ASO, Souza LCM, Borges HL. Highlights in Resistance Mechanism Pathways for Combination Therapy. Cells. 2019;8(9).

48. Le Tourneau C, Delord JP, Goncalves A, Gavoille C, Dubot C, Isambert N, et al. Molecularly targeted therapy based on tumour molecular profiling versus conventional therapy for advanced cancer 
(SHIVA): a multicentre, open-label, proof-of-concept, randomised, controlled phase 2 trial. The Lancet Oncology. 2015;16(13):1324-34.

49. Lee J, Kim ST, Kim K, Lee H, Kozarewa I, Mortimer PGS, et al. Tumor Genomic Profiling Guides Patients with Metastatic Gastric Cancer to Targeted Treatment: The VIKTORY Umbrella Trial. Cancer discovery. 2019;9(10):1388-405.

50. Boumahdi S, de Sauvage FJ. The great escape: tumour cell plasticity in resistance to targeted therapy. Nature reviews Drug discovery. 2020;19(1):39-56.

51. Kawazoe A, Fukuoka S, Nakamura Y, Kuboki Y, Wakabayashi M, Nomura S, et al. Lenvatinib plus pembrolizumab in patients with advanced gastric cancer in the first-line or second-line setting (EPOC1706): an open-label, single-arm, phase 2 trial. The Lancet Oncology. 2020;21(8):1057-65.

52. Fukuoka S, Hara H, Takahashi N, Kojima T, Kawazoe A, Asayama M, et al. Regorafenib Plus Nivolumab in Patients With Advanced Gastric or Colorectal Cancer: An Open-Label, Dose-Escalation, and Dose-Expansion Phase Ib Trial (REGONIVO, EPOC1603). J Clin Oncol. 2020;38(18):2053-61.

53. Li J, Qin S, Xu R, Yau TC, Ma B, Pan H, et al. Regorafenib plus best supportive care versus placebo plus best supportive care in Asian patients with previously treated metastatic colorectal cancer (CONCUR): a randomised, double-blind, placebo-controlled, phase 3 trial. The Lancet Oncology. 2015;16(6):619-29.

54. Al-Salama ZT, Syed YY, Scott LJ. Lenvatinib: A Review in Hepatocellular Carcinoma. Drugs. 2019;79(6):665-74.

\section{Figures}


A
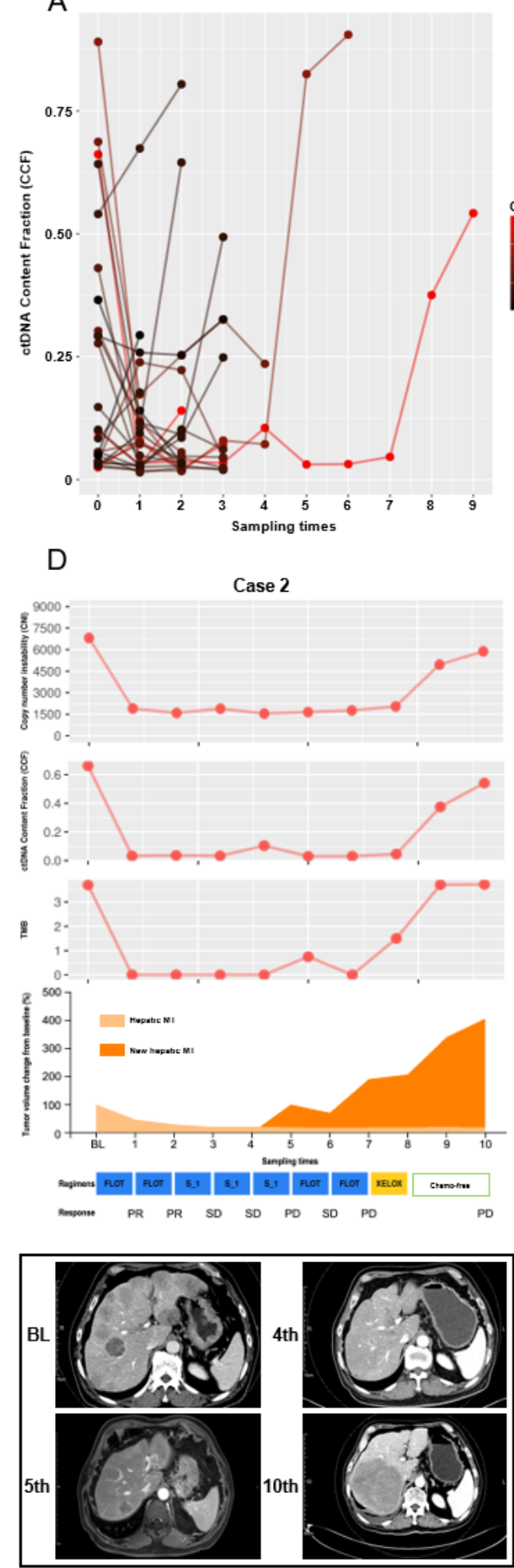

B
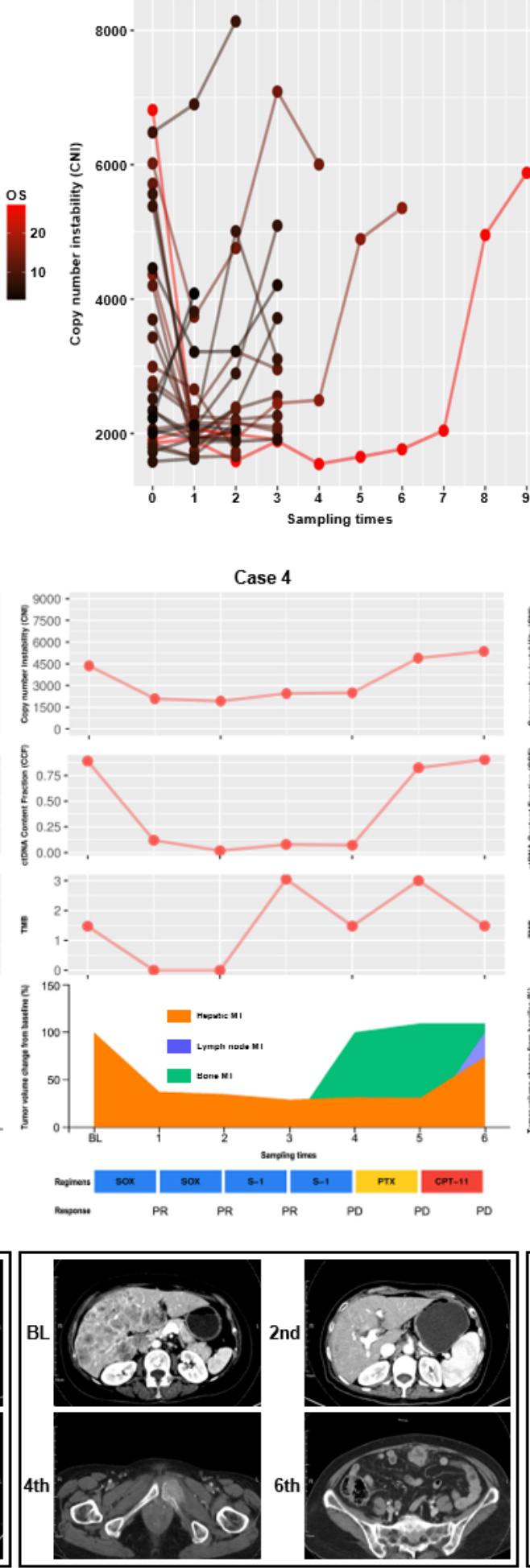

C
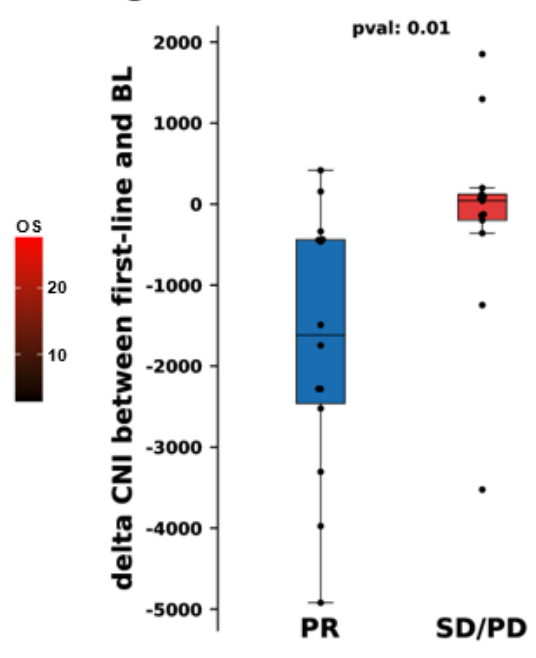

Case 17
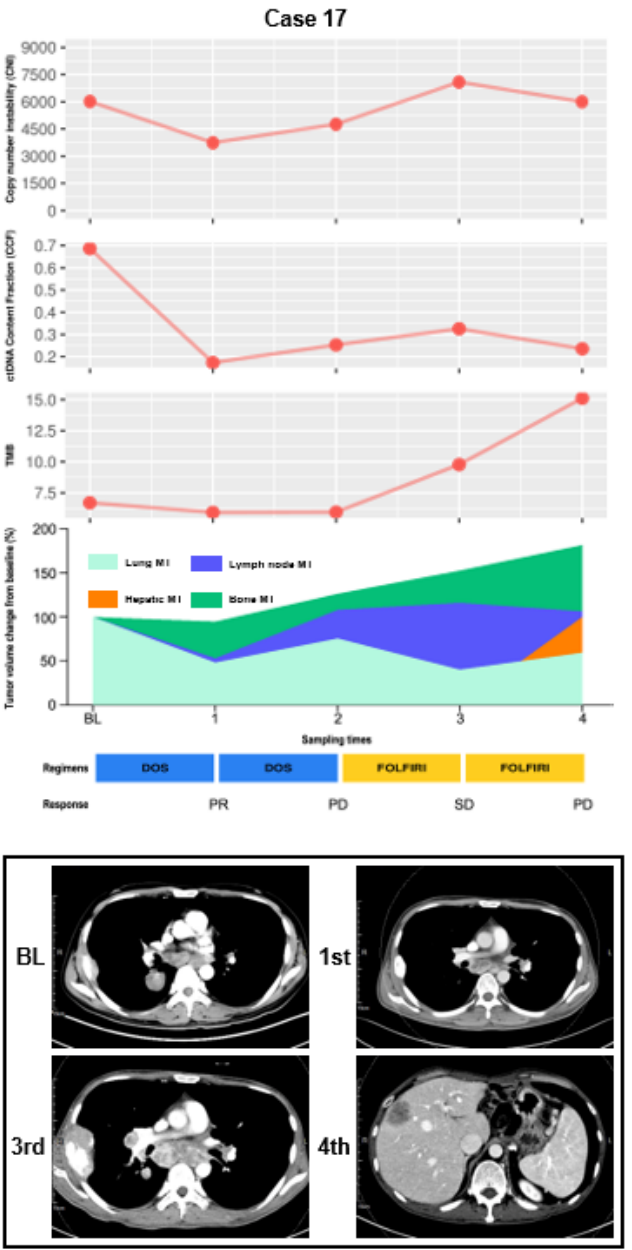

Figure 1

Dynamic change of genomic alterations during whole course treatment of patients. (A) The dynamic change of CCF during treatment of patients. (B) The dynamic change of CNI during treatment of patients. (C) CNI change between baseline and firs time of sampling correlated with tumor response. (D)Data of three typical cases during treatment was illustrated including CNI, CCF, TMB and tumor volume changes during treatment. 


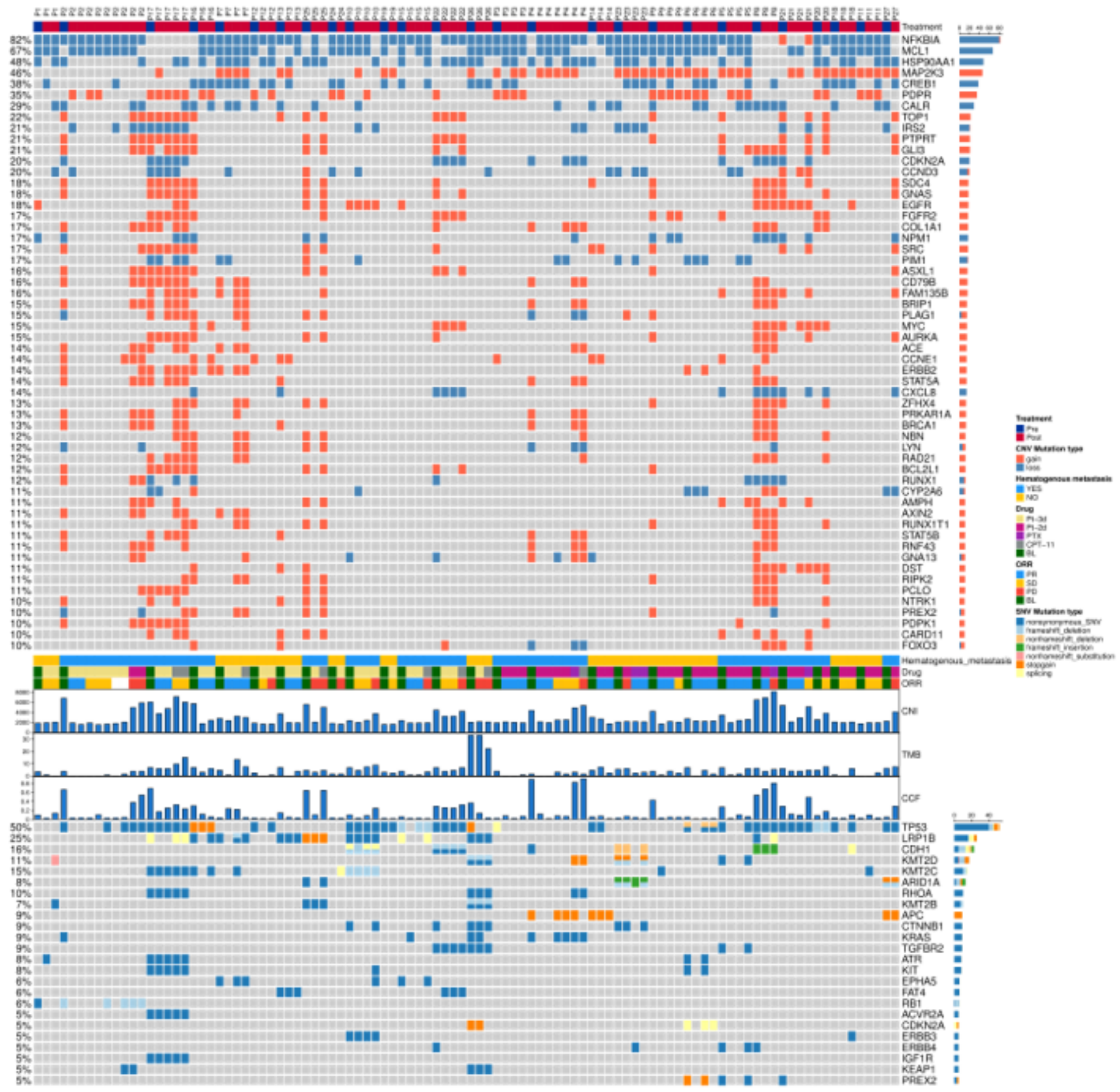

Figure 2

Landscape of genomic alterations of all samples. Gene-level CNVs and SNVs with high frequency of all patients were illustrated. 


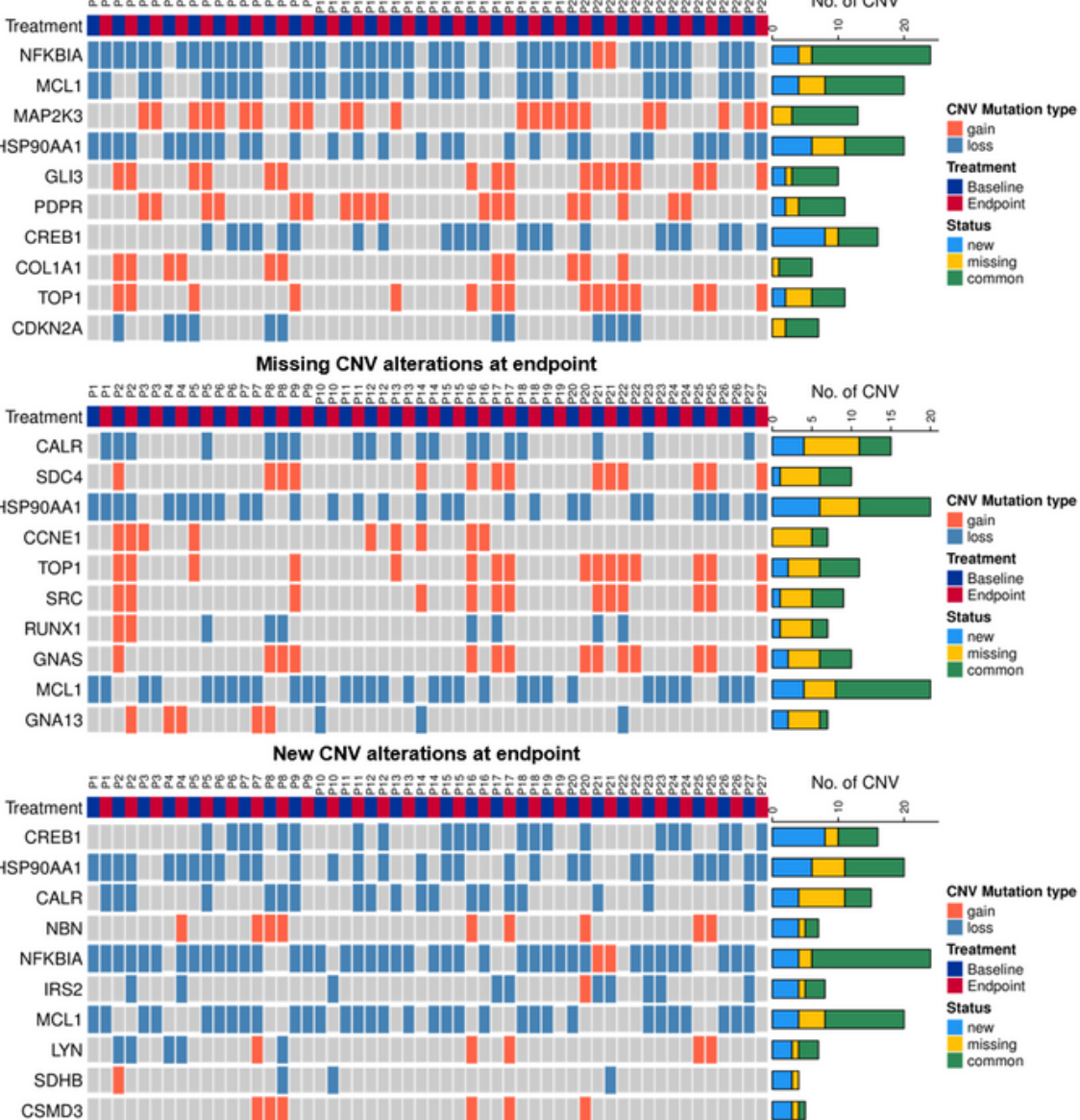

B

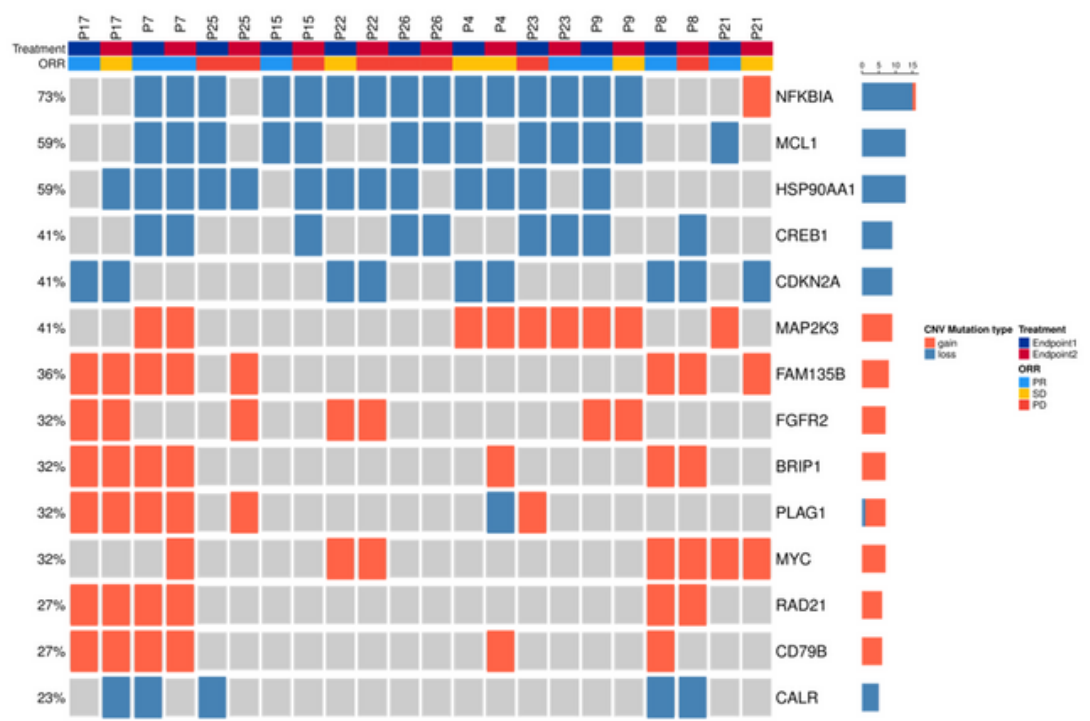

Figure 3

Gene-level CNV alterations during sequential treatment (A) Gene-level CNV alterations (CNV gain and loss) of all patients were compared between baseline and endpoint. Three categories of genes were classified. Proportion of three categories of genes were calculate and ranked in descending order. (B) Gene-level CNV alterations (CNV gain and loss) of 11 patients who received subsequent therapy were compared between EP1 and EP2. 

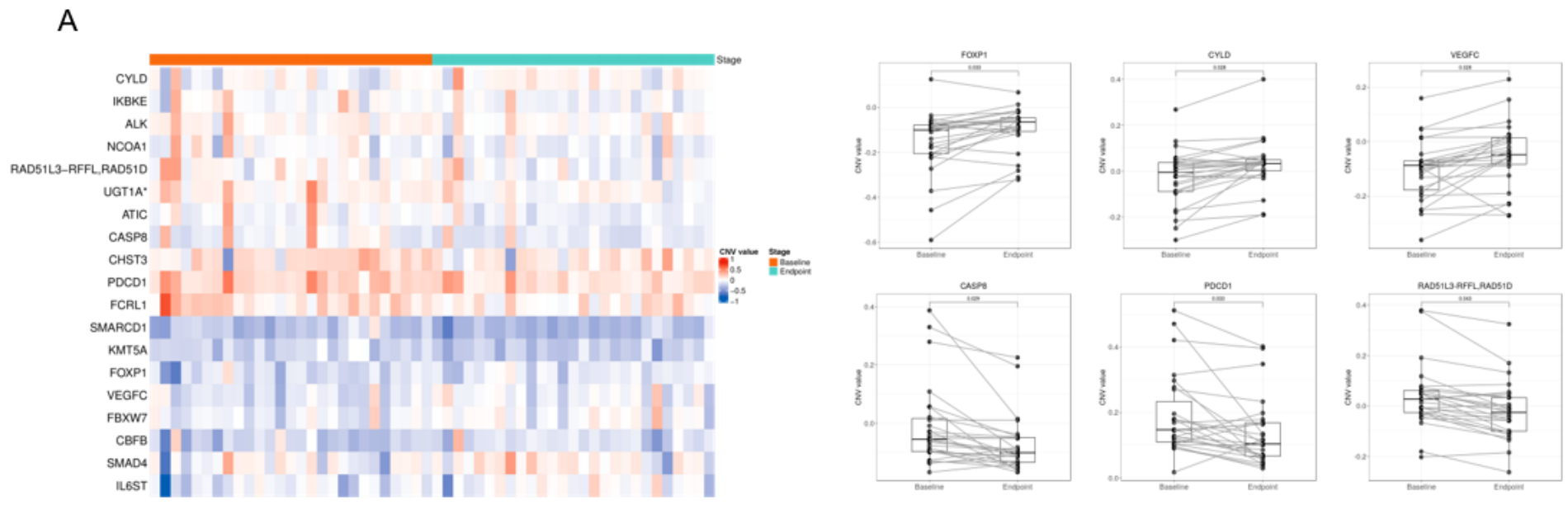

B
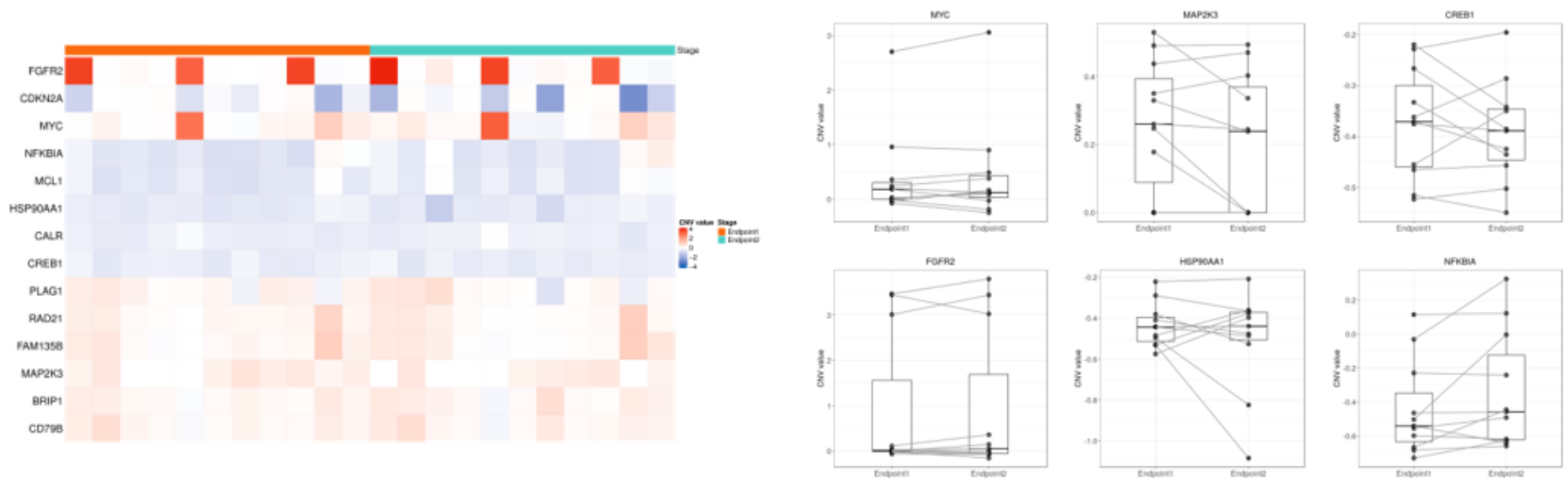

\section{Figure 4}

Changes of copy number values of genes during sequential treatment (A) Genes with differential copy number values in baseline and endpoint samples. (B) No significant difference of copy number values of genes was observed between EP1 and EP2 samples 
A

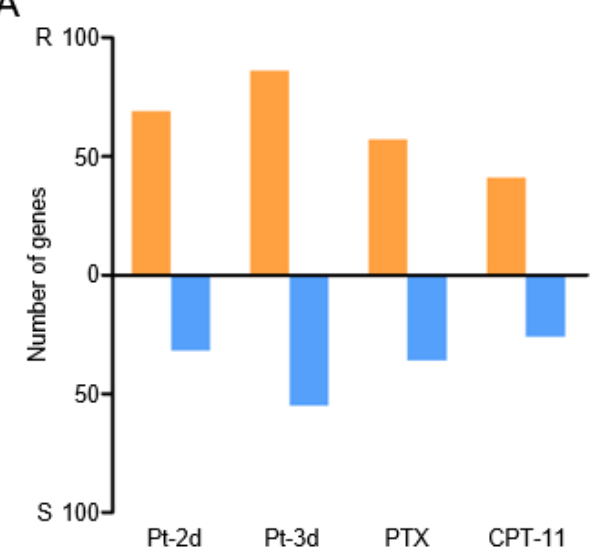

B

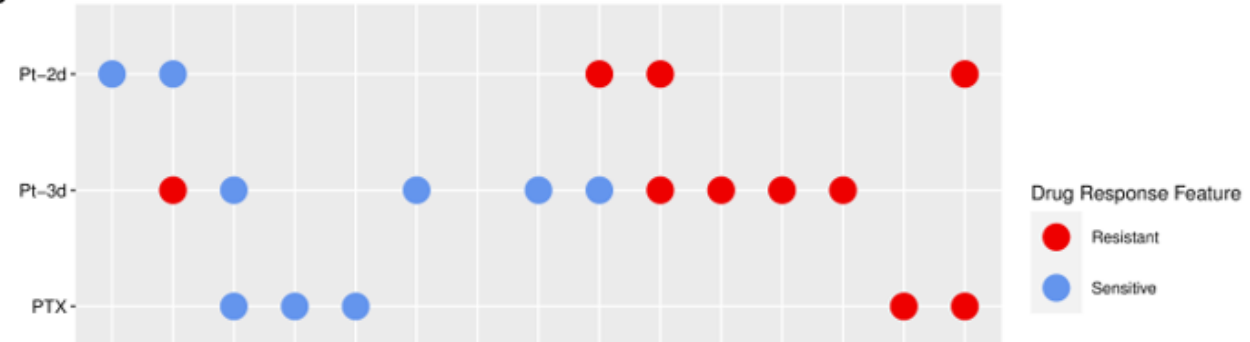

C

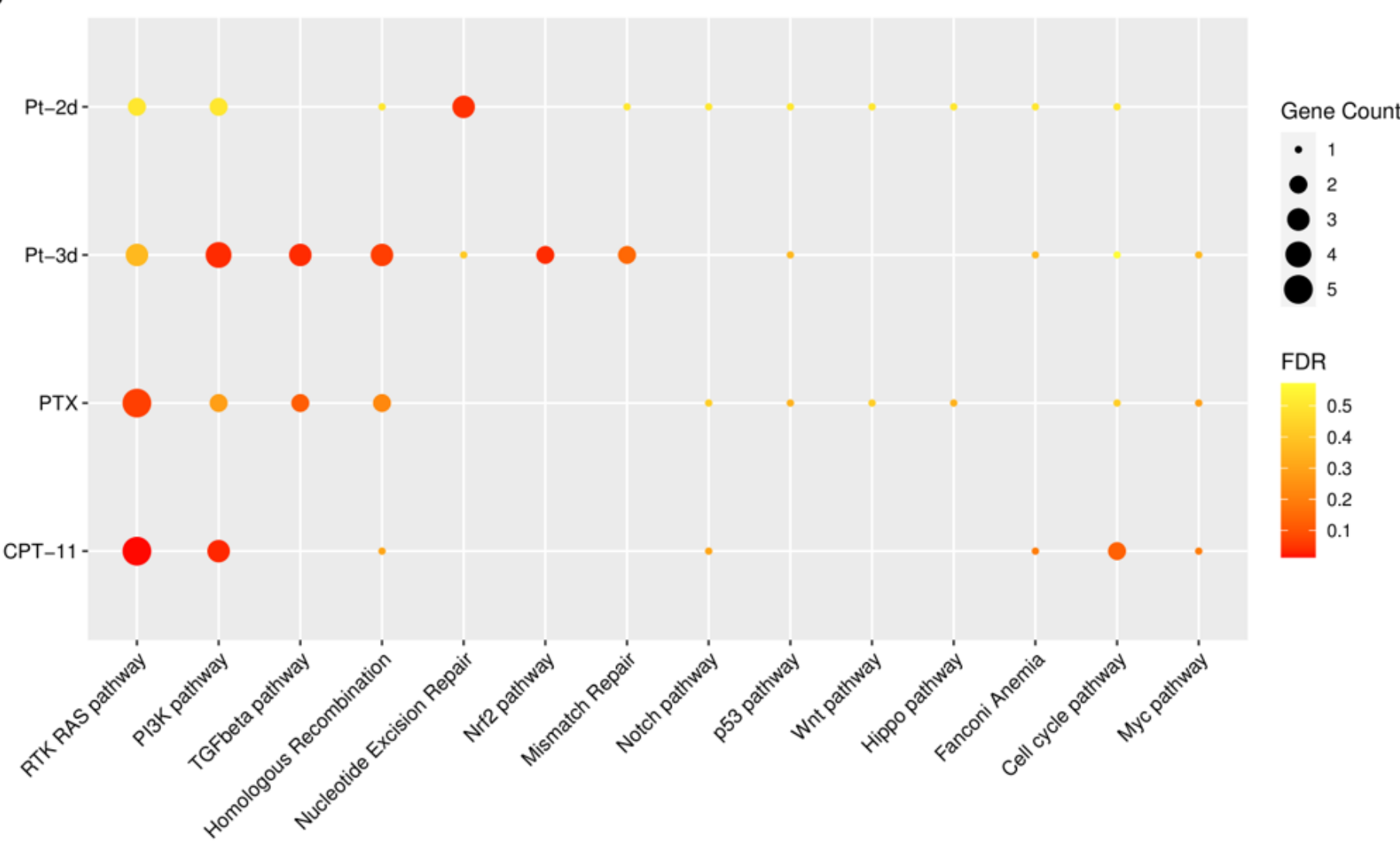

\section{Figure 5}

Chemotherapy regimens resistance related gene identification and oncological pathway enrichment (A) Number of resistance- and sensitivity-related genes identified in four regimens. (B) Genes with differential $\mathrm{CNV}$ values were defined as resistance- or sensitivity-related genes of regimens. (C) Oncological signaling pathway enrichment of resistance-related genes of four regimens. 
A

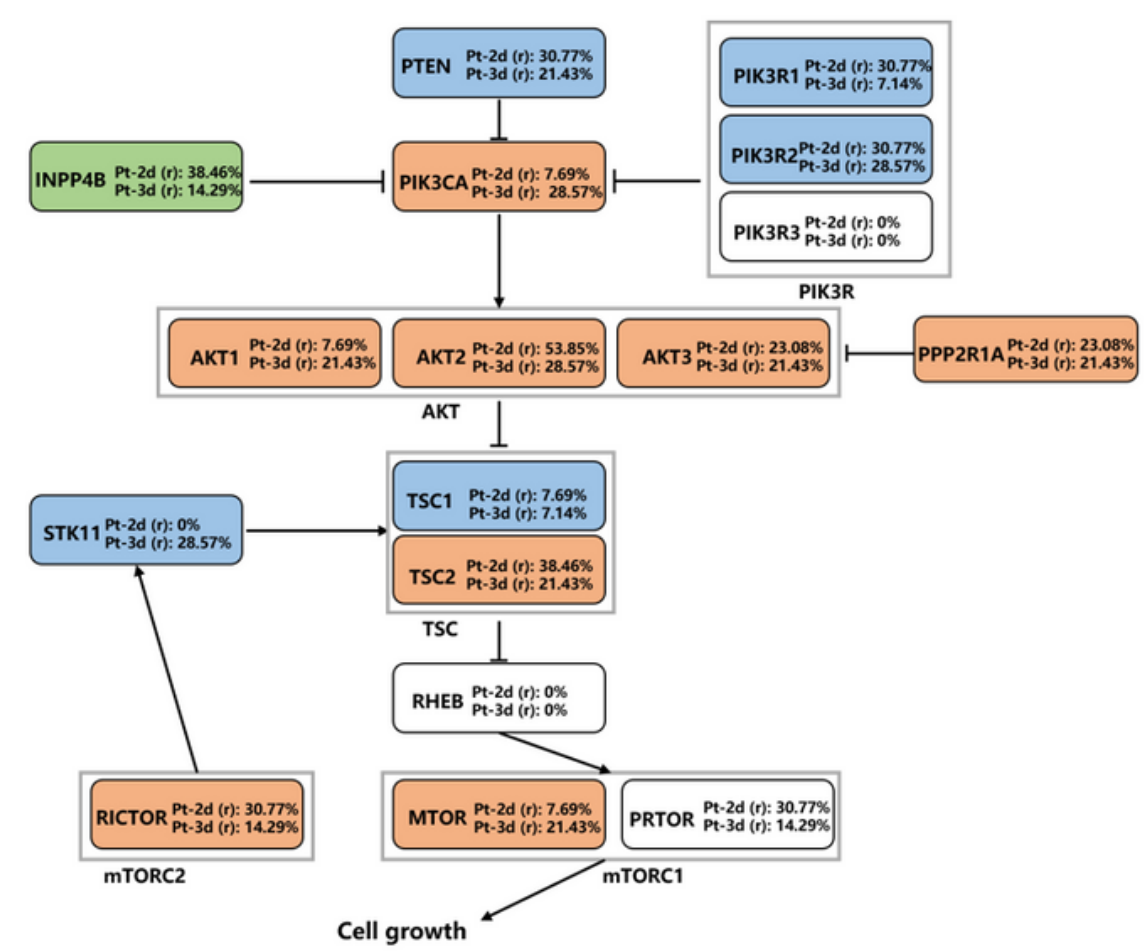

B

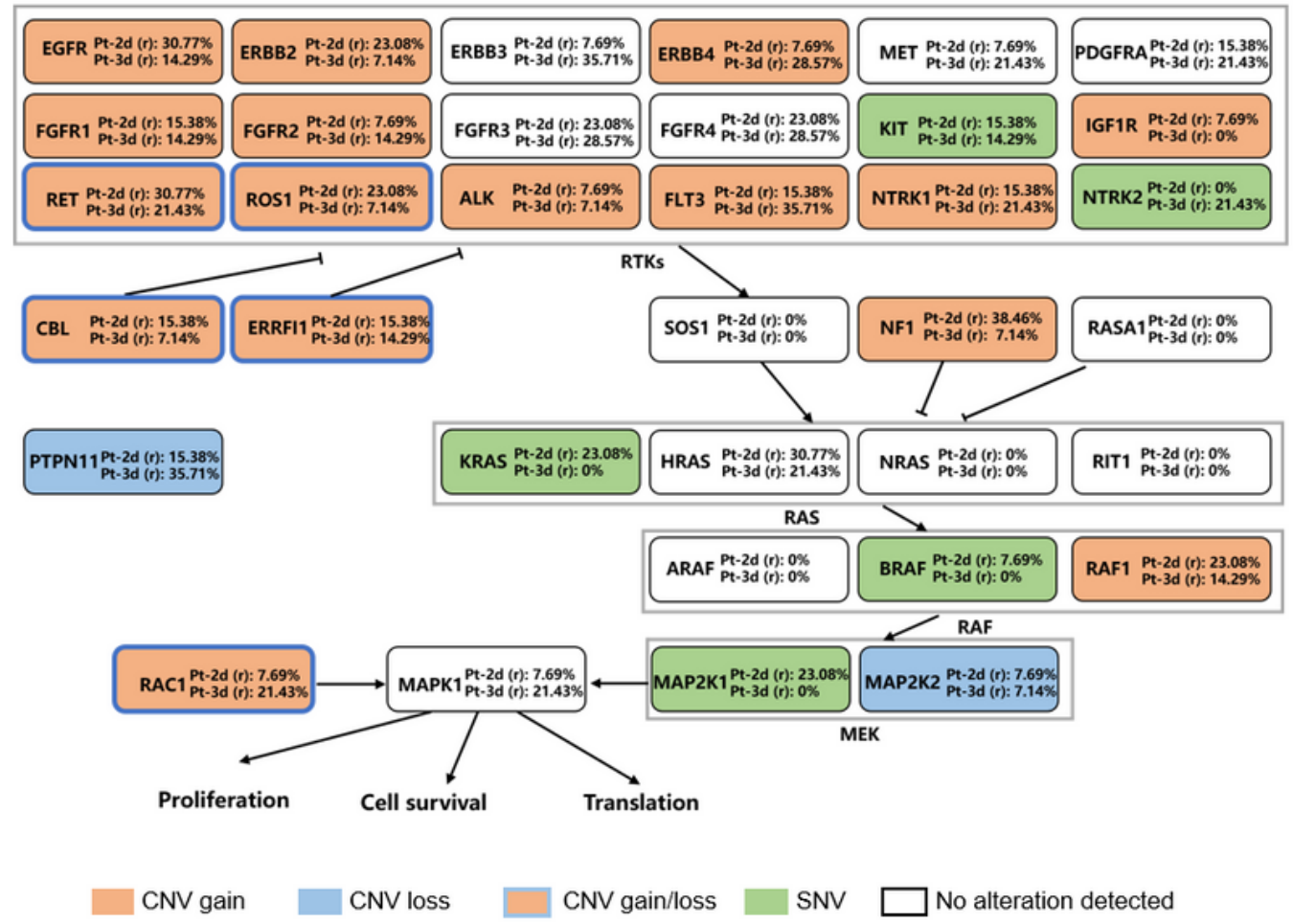

\section{Figure 6}

Resistance related genes involved in RTK RAS pathway and PI3K pathway (A) Genes in RTK RAS pathway were illustrated and labelled by genomic alterations detected at baseline and percentage of detected patients. (B) Genes in PI3K pathway were illustrated and labelled by genomic alterations detected at baseline and percentage of detected patients. 


\section{Supplementary Files}

This is a list of supplementary files associated with this preprint. Click to download.

- supplementaryfiles.pdf 